\title{
EL MOVIMIENTO \\ DE LA REFORMA \\ UNIVERSITARIA. \\ TRAYECTORIAS Y \\ TRASCENDENCIAS
}

Patricia

Funes

\section{(1) patfunes@gmail.com}

Universidad de

Buenos Aires/CONICET

Buenos Aires, Argentina

The University Reform movement. Trajectories and transcendences

\begin{abstract}
The reformist youth movement that emerged at the National University of Córdoba in 1918 was the instigator of a political-academic culture of the Argentine Public University. A matrix interpellated, discussed, resignified polyphonically according to the challenges of the times in the last hundred years. It also had an explicit Latin American projection, interlocution between "new generations" that made the movement a fertile territory to think about the region in the critical climate of the 1920s. The aim of the article is to analyze this reformist scene on three levels: at the institutional level and in the distribution of power at the Universities and at the level of beliefs and transcendences. In the latter we will explore the Latin American projection of the Reformism movement building some bridges between the 1920s and the 1960s.
\end{abstract}

\begin{abstract}
Resumen
El movimiento juvenil reformista surgido en la Universidad Nacional de Córdoba en 1918 fue instituyente de una cultura políticoacadémica de la Universidad Pública argentina. Una matriz interpelada, discutida, resignificada polifónicamente conforme a los desafíos de los tiempos en los últimos cien años. También tuvo una explícita proyección latinoamericana, interlocución entre "nuevas generaciones" que convirtió al movimiento en un territorio fértil para pensar la región en el clima crítico de la década de 1920. La intención del artículo es analizar esa escena reformista en tres niveles: en el nivel institucional y en la distribución del poder en el interior de las Casa de Altos estudios en Argentina y en el nivel de las creencias y trascendencias. En este último exploraremos la proyección latinoamericana de la Reforma, tendiendo algunos puentes entre los años veinte y los años sesenta.
\end{abstract}


I movimiento juvenil reformista surgido en la Universidad Nacional de Córdoba (UNC) en 1918 fue instituyente de una cultura político-académica de la Universidad Pública argentina. Una matriz interpelada, discutida, resignificada polifónicamente conforme a los desafíos de los tiempos en los últimos cien años. También tuvo una explícita proyección latinoamericana, interlocución entre "nuevas generaciones" que convirtió al movimiento en un territorio fértil para pensar la región en el clima crítico de la década de 1920.

Hacia 1918 en la entonces tricentenaria UNC la intransigencia de las autoridades frente a una demanda puntual de los alumnos produjo una radicalización del movimiento estudiantil que si en principio demandaba el recambio de las viejas Academias vitalicias en el gobierno de la Universidad (asunto que ya había ocurrido en la Universidad de Buenos Aíres entre 1906/1907 sin conflictos) llevó a la impugnación en bloque de sentidos, contenidos y misiones de las casas de Altos Estudios.

Los estudiantes de la "Casa de Trejo" portaban sus críticas: la omnipresencia de la Iglesia, la cooptación de la Universidad por parte de académicos y profesores del cerrado círculo de "familias patricias", la misión restringida de las universidades dedicadas exclusivamente a la expedición de títulos profesionales, las restricciones en el ingreso a la Universidad. También, una crítica a los paradigmas gnoseológicos: para algunos las autoridades tomistas, para otros "la mano áspera de la filosofía positiva" que inhibía humanidades y éticas.

La intención del artículo es analizar esa escena reformista en tres niveles: en el nivel institucional, en la distribución del poder en el interior de las Casa de Altos estudios en Argentina y en el nivel de las creencias y trascendencias. En este último exploraremos la proyección latinoamericana de la Reforma, tendiendo algunos puentes entre los años veinte y los años sesenta. ${ }^{1}$

\section{Contextos e Instituciones}

La institución no es tan sólo muros y estructuras exteriores que rodean, protegen, garantizan o constriñen la libertad de nuestro trabajo, sino que es también la estructura de nuestra interpretación. (DERRIDA, 1984, p. 45).

Hacia 1918 en Argentina existían tres Universidades nacionales: Córdoba (UNC), creada en 1613, Buenos Aires (UBA), en 1821 y La Plata (UNLP), en 1905. Dentro del formato napoleónico prevaleciente, Córdoba era “monástica y monárquica”, Buenos Aires, liberal y positivista y La Plata moderna, científica. La universidad-convento, la universidad-bufete y la universidad-laboratorio (ROJAS, 1915, p. 92), cumplían con la dotación de profesionales para el Estado y la economía en expansión, reclutados en el estrecho círculo de las elites oligárquicas.

Si se revisan los miembros de los tres poderes del Estado a nivel nacional y provincial, se advierten vasos comunicantes directos con académicos y autoridades de las Casas de Altos Estudios. El caso de Nicolás Avellaneda que fue rector de la 
UBA (1881-1885), paralelamente senador nacional por Tucumán y autor de la primera ley universitaria (Ley 1.597 denominada “Ley Avellaneda”,1885) luego de haber ejercido la Presidencia de la República (1874-1880), aunque excepcional, formaba parte de una dinámica común. Vayan sólo tres ejemplos: en Córdoba, José Antonio Ortiz y Herrera, miembro de la Venerable Orden Tercera Franciscana, fue decano de la Facultad de Ciencias Médicas (1890-1894), rector de la UNC casi diez años (18971907) y dejó el decanato para asumir la gobernación de la provincia. Paralelamente fue senador provincial (1886-1891) y vicegobernador de la provincia (1895-1898). Norberto Piñero formó parte de la Comisión redactora del Código Penal argentino, junto con Nicolás Matienzo y Rodolfo Rivarola (compañeros de la promoción de la Facultad de Derecho de la UBA del año 1882)². En dos oportunidades Piñero renunció al decanato de la recientemente creada Facultad de Filosofía y Letras de la UBA para ser Ministro de Hacienda, convocado por los presidentes José Figueroa Alcorta (1906) y Roque Sáenz Peña (1912). ${ }^{3}$ En el caso de La Plata, Joaquín V. González (1863-1923), destacado hombre de letras, periodista, jurista, político, en 1901 fue Ministro del Interior (designado por Julio Argentino Roca), sin embargo mantuvo su cátedra de Derecho en la UBA. El presidente Manuel Quintana lo designó Ministro de Justicia e Instrucción Pública en 1904. En ese cargo fue un activo promotor de la creación y nacionalización de la UNLP (1905) de la que fue rector (presidente, en la normativa de la nueva universidad) durante 12 años (1906-1918). Consignamos estos ejemplos para poner en evidencia, alguno de los significados asociados a la demanda de "autonomía universitaria" respecto de esos académicos que en el caso de Córdoba eran escogidos atravesando "el triple cernidor del abolengo, de la política y de la religión”, como señalaba el presidente de la Federación Universitaria de Córdoba, Enrique Barros. ${ }^{4}$ (BARROS, 1918, p. 11)

El acceso al cargo por concurso, la periodicidad de cátedra y los cursos de docencia libre demandados por los estudiantes, contribuían a demarcar perímetros de lo estrictamente universitario, al tiempo que despatrimonializaba y modernizaba el claustro docente y, en gran medida, la elección de autoridades. La crítica a la "fábrica de títulos" y al profesionalismo de las universidades iba acompañada de una concepción menos utilitarista de la Universidad. La exigencia de creación y no mera repetición de conocimientos supuso la expansión de centros e institutos de investigación, a partir de los cuales ingresaron a la Universidad profesores más jóvenes y más plebeyos. Por ejemplo el reformista José Babini, matemático, químico, historiador de la ciencia, hijo de inmigrantes, trabajó desde los doce años como cadete en una empresa constructora que lo apoyó económicamente para cursar sus estudios y a los veintitrés años era decano interino de la Facultad de Ingeniería Química de la nueva Universidad Nacional de Litoral. En los años sesenta sería decano de la Facultad de Ciencias Exactas de la UBA (1955-1957), organizador y rector interino de la Universidad Nacional del Nordeste (1957) y al año siguiente, vicerrector y primer presidente del directorio de la Editorial Universitaria de Buenos Aires (EUDEBA). Fuera del espacio reformista, y de trayectoria excepcional, Bernardo Houssay ingresó a la Carrera de Farmacia de Medicina de la UBA a los catorce años, se graduó a los diecisiete y a los veintitrés años se recibió de médico. Pudo completar sus estudios porque la UBA 
lo eximió del pago de los aranceles. En 1919 accedió a la cátedra de Fisiología de la Facultad de Medicina de la UBA y organizó el Instituto de Fisiología de la Facultad (fue Premio Nobel de Medicina en 1947). En el campo de las humanidades, se incorporaron por concurso nuevos profesores, innovadores todos en sus respectivas disciplinas: Emilio Ravignani y Salvador Debenedetti en los años veinte son directores de dos de los cinco Institutos de Investigación de la Facultad de Filosofía y Letras (Instituto de Investigaciones Históricas y Museo Etnográfico. Otro tanto Roberto Giusti o Coriolano Alberini, destacados intelectuales en las letras y la filosofía, ostensibles portadores todos de apellidos inmigrantes.

Las tres universidades tenían culturas institucionales diversas y autocentradas, con muy pocas relaciones entre sí. El reformismo quebró ese aislamiento. Por un lado expandió el paisaje universitario: se nacionalizaron las Universidades del Litoral y de Tucumán. ${ }^{5}$ Ambas con formatos novedosos territoriales y disciplinarios, no exentos de críticas que escondían el delicado tema de la competencia por la acreditación de títulos a nivel nacional y la expansión del presupuesto. Ricardo Rojas, intelectual y académico defensor del reformismo, señalaba que criticar a la Universidad de Tucumán porque no tenía Facultad de Derecho era "como si en tiempos de Rivadavia se hubiera objetado la de Buenos Aires por no tener una Facultad de Teología.” (ROJAS, 1915, p. 96)

Los estudiantes lograron cohesionar un movimiento atravesando las cinco universidades a través de las federaciones estudiantiles provinciales y de la federación nacional (FUC, FUBA, FULP, FUA). Por otra parte, el reconocimiento de títulos habilitantes y competencias que otorgaba el Estado, contribuyó a crear un sistema universitario nacional más homogéneo y a la vez más plural. También fue la matriz de sociabilidades que trascendieron lo político y fueron vínculos y afectividades de larga data. Este rasgo también es fundacional del ethos de las universidades argentinas, sobre el que nos referiremos más adelante. Saltando en el tiempo en otro momento de recuperación del ideario reformista, el sociólogo Miguel Murmis en una entrevista que ya tiene sus años, se refería a sus tiempos estudiantiles en los años sesenta en el sentido antes indicado: "Era muy intensa la relación interfacultades (...). Toda la gente de mi generación va al dentista que era el Presidente del Centro de Estudiantes de Odontología. Ahí se daba la posibilidad de la comunidad (...). Fue también un descubrimiento del país: uno viajaba a Tucumán, a La Plata, a Córdoba por el tema de la FUA.”(MURMIS, 1988).

\section{Estudiantes y jóvenes: poder, autoridad, ciudadanía y democracia}

La juventud vive siempre en trance de heroísmo. Es desinteresada, es pura. No ha tenido tiempo aún de contaminarse. No se equivoca nunca en la elección de sus propios maestros. Ante los jóvenes no se hace mérito adulando o comprando. Hay que dejar que ellos mismos elijan sus maestros y directores, seguros de que el acierto ha de coronar sus determinaciones. Manifiesto Liminar, 1918. 
El canónico documento de la Reforma Universitaria, escrito por Deodoro Roca y publicado el 21 de junio de 1918 en el número 10 de La Gaceta Universitaria, se conoce hasta el día de hoy con el nombre de Manifiesto Liminar. ${ }^{6}$ Sin embargo, llama la atención que su título original no hiciera alusión a ningún asunto estrictamente universitario: La Juventud Argentina de Córdoba a los hombres libres de Sudamérica. Manifiesto de la Federación Universitaria de Córdoba. La autoidentificación no es la de estudiantes sino la de jóvenes. El juvenilismo es un tropo trascendente de la época: lo joven, lo nuevo, incluso lo heroico forman parte de las revisiones paradigmáticas de la primera posguerra. Esa juventud no es un rango etario o biológico sino que se identifica por un imperativo moral que deviene político. A partir del péndulo de las generaciones ortegueanas entre épocas cumulativas y épocas eliminatorias (ORTEGA Y GASSET, 1947, p. 18) la adscripción reformista a una "nueva generación" acompaña una "nueva sensibilidad" portadora de valores políticos, sociales, éticos y estéticos diferenciados de sus predecesores.

El continente juvenil expresaba con mayor precisión que la condición de estudiantes, ese actor social en el que se reconocían los impulsores de un conjunto de reclamos que trascendían los claustros universitarios: es el país, no solo es la universidad la que cuenta con "una vergüenza menos y una libertad más" a partir de la acción estudiantil. La juventud, más allá de las condiciones universitarias, supone esa nueva sensibilidad contestataria, de lo contrario "es una simple vejez que se anticipa a las canas", como señalara el referente e ideólogo intelectual José Ingenieros. (INGENIEROS, 1927, p. 380). De allí la interpelación genérica que titula el Manifiesto Liminar y recorre el movimiento en los años veinte. Podría argüirse además que algunos de los líderes cordobeses, por caso, el mismo Deodoro Roca o Arturo Capdevila, que no firman el documento, ya estaban graduados, claustro para el cual también se prevé una representación en el cogobierno universitario.

Esos jóvenes hicieron suyas herramientas de espacios periféricos y subalternos con los que tenían relaciones precedentes. Desde el punto de sus formaciones, con grupos intelectuales fuera de una Universidad poco estimulante para vocaciones inquietas (vgr. Asociación Córdoba Libre). ${ }^{7}$ Desde el punto de vista de las alianzas sociales: con el movimiento obrero (los ferroviarios, los trabajadores del calzado, los molineros, los empleados municipales). La articulación entre cenáculos intelectuales alternativos, el periódico o la revista, los congresos, la huelga y las manifestaciones callejeras como mecanismo de presión, fortalecieron y dieron visibilidad a su programa a nivel nacional. No fue azaroso, entonces, que el primer número de La Gaceta Universitaria. Órgano de la Federación Universitaria de Córdoba, haya salido el día proletario mundial: 1 de mayo. El programa reformista fue adquiriendo contornos más precisos y ambiciosos enhebrando el adentro y el afuera de la Universidad a partir de los debates acerca de los modos de aprender y enseñar, reproducir y crear conocimiento en la Universidad y también revisar los presupuestos de su gobierno: la representación, la ciudadanía y el demos universitario.

Consideramos que la ruptura más importante del movimiento reformista de la década de 1920 es la que coloca a los estudiantes/jóvenes en el centro del hecho 
educativo y del gobierno de la universidad en diálogo y tensión con el conjunto de debates sobre la ampliación de la ciudadanía y los fundamentos de la democracia política, herederos de la victoria del yrigoyenismo a partir de 1916.

Las maneras de expresar impugnaciones y propuestas en el Manifiesto están en correspondencia con ese espíritu nuevo explícitamente enunciado en el documento fundacional: son "las resonancias del corazón", no las de la pura razón las que advertían que estaban "pisando una revolución, viviendo una hora americana." En el mismo registro se expresa el dispositivo pedagógico que reemplazara las "formas de tiranizar y de insensibilizar" dictadas en las cátedras de la UNC: un lazo espiritual y ético entre profesores y estudiantes fundado en una paideia que revisaba principios de autoridad y poder:

La autoridad en un hogar de estudiantes, no se ejercita mandando, sino sugiriendo y amando: Enseñando. Si no existe una vinculación espiritual entre el que enseña y el que aprende, toda enseñanza es hostil y de consiguiente infecunda. (...) En adelante solo podrán ser maestros en la futura república universitaria los verdaderos constructores de alma, los creadores de verdad, de belleza y de bien.

El Manifiesto se redactó en el momento en que fracasaba la solución reformista o gradualista de la intervención enviada por el Poder Ejecutivo Nacional para apoyar las demandas estudiantiles, o mejor, cuando las autoridades de la UNC se mantienen impermeables a la propuesta de democratización institucional que, entre otras reformas, preveía el reemplazo de los académicos vitalicios por los profesores de la Universidad. Los estudiantes ven así coartadas las posibilidades de materializar sus peticiones, toman el rectorado de la UNC y declaran la huelga general. En este clima de radicalización se elabora el Manifiesto. La declaración tiene, por un lado, un carácter político y denuncista que bien podría no haber trascendido el carácter episódico del que fue producto. Sin embargo, los alcances posteriores del movimiento de la Reforma Universitaria y, sobre todo, los contenidos, aunque generales, programáticos de sus postulados resignificaron la declaración de junio de 1918 otorgándole ese carácter "liminar."

En el momento que los profesores "traicionan" los consensos obtenidos y votan en la Asamblea del 15 de Junio como rector a Antonio Nores (conspicuo representante de la Corda Frates ${ }^{8}$ ), se agudizó el conflicto estudiantil. La impugnación a toda la "casta profesoral", resta legitimidad moral a aquellos que los mismos estudiantes habían propuesto para el gobierno de la universidad en reemplazo de las academias y exigen su derecho a cogobernar. La soberanía de ese reclamo se basa en la revolución moral que esgrimen: "la juventud ha sido capaz de realizar una revolución en las conciencias, no puede desconocérsele la capacidad de intervenir en el gobierno de su propia casa." La idea del cogobierno recupera por homologación la figura de la república democrática: "la Universidad es una República de Estudiantes”, señalaba Gabriel Del Mazo. "Son estudiantes todos los copartícipes en la Comunidad de estudios. El alumno, 
titular de los derechos cívicos en la Nación, toma con derecho, partiาcipación en la vida y gobierno de esta república menor" (DEL MAZO, 1946, p. 9)

La democracia universitaria estaba lejos de tener contornos definidos. Propagado rápidamente el movimiento en las cinco universidades del país, se debatió en el Primer Congreso Nacional de Estudiantes la normativa que reemplazaría la Ley Avellaneda que hasta entonces regía a las Universidades. ${ }^{9}$ Esa "república menor" sería gobernada por "todos los que pertenezcan a ella: estudiantes, diplomados y profesores. "Es de la esencia de una república democrática que, cuando su gobierno esté organizado de acuerdo al sistema representativo, participen todos los ciudadanos de la elección de las autoridades." (DEL MAZO, 1946, p. 11)

El demos universitario "se asentó en el supuesto tácito de una relación virtuosa entre democracia, saber y autoridad donde el primer término se planteaba como expresión de la participación de todos los claustros que basa su autoridad no solo en la representatividad sectorial sino también en el reconocimiento de un saber o competencia legítima. (SUASNABAR, 2006, p. 57). Sin embargo, más allá de la significativa ampliación de la ciudadanía universitaria, la representación asumía una cualidad específica para evitar que en los cuerpos directivos se diera el predominio de cualquiera de los "tres estados" (profesores, estudiantes y diplomados o profesionales):

Hemos convenido llamar "estados" a los diferentes cuerpos de estudiantes, profesores y profesionales, por cuanto la soberanía universitaria reside en el concurso de esos núcleos cuyos derechos no son proporcionales al número de sus componentes. El sufragio no es proporcional al número de sufragantes, siendo la representación fija e invariable. Se establece por este medio, no la democracia del número, sino la legítima y proporcional representación de los intereses."(WATSON, 1926, p. 29)

En esta y otras formulaciones acerca de la representación y la ciudadanía se advierte tanto el repudio a la "vieja política criolla" oligárquica cuanto a las formas de demoliberales de representación instaladas en el país a partir de la Ley Sáenz Peña de sufragio universal (un ciudadano, un voto) ensayadas por primera vez en las elecciones de 1916. La solución de los estudiantes está mucho más cerca del concepto "democracia funcional", centro temático y teórico para el gobierno de "los tiempos nuevos" defendida por José Ingenieros. José Ingenieros sintetiza, cuando no traduce y divulga, en sus escritos muchas de esas referencias. En La Universidad del porvenir, aparecido inicialmente en 1916 problematiza núcleos neurálgicos del ideario reformista del 18 tales como "exclaustración de la cultura", las Universidades Populares, la representación estudiantil. Pero será su idealismo moral, el concepto clave de esas "fuerzas morales" a las que convoca Ingenieros y que retoman los reformistas cordobeses con gran fidelidad." Todas estas características resultan notorias ya en el Manifiesto Liminar (...), donde -en términos notablemente ingenierianostambién se afirma el derecho de las fuerzas morales contra 'los intereses creados en torno de los mediocres'." (TERAN, 1979, p. 109) Ecos del pensamiento de Ingenieros también se escuchan en el planteo meritocrático, elitista y de vanguardia misional, tan 
frecuente en las proclamas juveniles. Por otra parte en el libro El hombre mediocre propone dotar de legitimidad de las minorías ilustradas en la dirección del país y en el proceso constitutivo de la Nación. Desde esta argumentación Ingenieros se opuso explícitamente al sufragio universal. ${ }^{10}$

La relación entre universidad y democracia fue planteada en el Congreso antes citado por Emilio Biagosh. Consideramos que ese texto es representativo de las tensiones, las búsquedas y las no siempre lineales relaciones entre la democracia en el país y en la universidad. Para el delegado oficial de la FUA la universidad es el instrumento privilegiado para la realización y la profundización de la democracia. Discute la idea de "crisis de la democracia" en el país (presente en los discursos conservadores a partir de 1916) al tiempo que señala el anacronismo de funciones y valores universitarios para fortalecerla. Para cumplir esa misión "reclama el máximo de libertad y soberanía" en relación al poder político: "Concretando este sería mi proyecto de ley: artículo único. Destínase al sostenimiento de las universidades nacionales e institutos de alta cultura el $6 \%$ de las rentas generales de la nación." (BIAGOSH, 1927, p. 110). Todo lo demás debería ser resuelto y decidido por los actores universitarios: "gobierno, planes de estudio, métodos de enseñanza, sistema de promoción, etcétera. En nada de eso deberían intervenir los poderes del Estado."

La relación universidad-democracia discurre por dos centros temáticos. Por un lado la autonomía de la Universidad respecto del Estado (legitimidad y soberanía de los estudiantes para participar en el gobierno universitario, formas de representación, formación cívica para ejercer esa ciudadanía) y hacia afuera de la Universidad dialoga y debate sobre la relación entre la educación, la nacionalidad y los resultados de la ampliación de la ciudadanía política en el país. La disertación de Biagosh aborda todos los niveles educativos (desde la escuela primaria a la Universidad) y se detiene, incluso, en la educación de las mujeres. ${ }^{11}$

Cita a Saúl Taborda -a quien califica "uno de los espíritus vanguardias de Córdoba"para tomar el diagnóstico que éste hace sobre la relación Universidad-democracia. Para Taborda las universidades no han sido capaces de democratizarse sólo se han modernizado, la democracia americana no se concreta a ser una función electoral; la comprende como comprende también a la justicia, a la propiedad, a la educación, a la religión, al arte y a la moral."(BIAGOSH, 1927, p. 100). Biagosh cita prudentemente a Taborda, quien sostenía posiciones más virulentas para exponer su concepción de la democracia y soberanía deudoras de un sustrato comunitarista y funcionalista que es previa a la función electoral.

Las relaciones entre los estudiantes y el Poder Ejecutivo Nacional emanado de las primeras elecciones que sanearon y ampliaron a través del voto secreto, obligatorio y "universal" las prácticas y contenidos políticos fueron de complementariedad, no de mímesis. Cabe recordar que a pesar de la reafirmación de la autonomía universitaria los estudiantes cordobeses solicitan la intervención del Poder Ejecutivo en dos ocasiones y que el Ministro de Justicia e Instrucción Pública, José Santos Salinas, fue una suerte de embajador del yrigoyenismo que apoyaba las demandas estudiantiles en las universidades. Ambos, movimiento estudiantil y autoridades nacionales convergían 
en la necesidad de superación del orden oligárquico y en el caso de los jóvenes/ estudiantes llamaban a pensar la política y lo político a partir de esa experiencia que llamaban "revolucionaria" en el entendimiento que la Universidad era el lugar para profundizar esa democracia incompleta. A nuestro juicio se expresan, tanto la vocación dirigente de la "nueva generación" cuanto la confianza taumatúrgica en el poder de la educación para transformar la sociedad y la política, continuidad iluminista que heredan y resignifican desde la tradición sarmientina.

\title{
Creencias y trascendencias. América Latina
}

\begin{abstract}
"Andamos por la tierra de América, sin vivir en ella. Las nuevas generaciones empiezan a vivir en América, a renegar de literaturas exóticas, a medir su propio dolor, a poner alegría en la casa, con la salud y con la gloria de su propio corazón" (DEODORO ROCA. 1918)
\end{abstract}

El excepcional latinoamericanismo del movimiento se expresa de manera diáfana desde el título del Manifiesto. En ocasiones se naturaliza ese rasgo fundacional del reformismo, singularidad y novedad en la tradición "europeísta” de la cultura argentina La interpelación a "los hombres libres de Sudamérica”, puede reconocerse en la oposición "república libre- dominación monástica y monárquica” del primer párrafo, inspirada en el pensamiento de la emancipación y en el primer liberalismo jacobino que la acompañó. La medida continental de la gesta emancipadora, por ende, acompaña esa invocación y es una ruptura antihegemónica que instala valores alternativos al orden de ideas prevaleciente.

El tema latinoamericano reconoce también una deuda con la generación modernista del novecientos: José Enrique Rodó y Manuel Ugarte, cada uno a su modo, echan las bases de una problematización sobre la región que recogen los jóvenes cordobeses. El Ariel, publicado en 1900 (a dos años de la guerra americano-española que termina con los últimos dominios de la corona de España en América) expresó conceptos que los intelectuales latinoamericanos estaban muy dispuestos a escuchar. (FRANCO, 1985, p. 65). Haciendo suya la metáfora shakespereana recreada por Renan plantea en términos dicotómicos una América Latina espiritual e idealista (Ariel) enfrentada a unos EEUU pragmáticos y materialistas (Calibán). Con esa operación impulsaba la unidad por la diferencia y la comunidad cultural de América Latina. Por su parte, Manuel Ugarte, otro "Maestro de la Juventud" dedicó numerosos escritos a la prédica por la unidad latinoamericana. En El Porvenir de América sintetizaba su ideario de unidad continental, que acompañó con un recorrido bolivariano entre 1911 y 1913 por una veintena de países de la región, haciendo pública su voluntad de construir "el andamiaje de un sistema de defensa continental contra el imperialismo anglosajón." Manuel Ugarte, al igual que el socialista Alfredo Palacios, prestó su apoyo al movimiento estudiantil, no sólo adhiriendo a sus proclamas sino también participando de sus actos y congresos. Ugarte, por ejemplo, fue el único orador no universitario en el acto de proclamación de la Federación Universitaria Argentina, el 11 de abril de 1918. 
Pero fueron los resultados de la Gran Guerra los que expresaron un clivaje en el que se astillan gran parte de las certezas y mayúsculas decimonónicas: "Civilización”, "Progreso", “Razón”, “Liberalismo”, "Positivismo”. La crisis postbélica crea nuevos objetos de pensamiento: la nación, las masas, el antiimperialismo, la revolución, el indigenismo, las vanguardias estéticas y políticas.

"No es que tengamos brújula propia, es que hemos perdido la ajena”, escribía Pedro Henríquez Ureña en la revista estudiantil Valoraciones (HENRIQUEZ UREÑA, 1978, p. 52). Si los "bárbaros" europeos se habían suicidado en una guerra, como proclamaba no sin desconsuelo José Ingenieros, el carácter de "civilización" podía ser revisado, incluso, invertido. Para José Vasconcelos, por ejemplo, la barbarie en la historia se había demostrado más creativa como matriz de las civilizaciones. La modernidad latinoamericana, entonces, podía juzgarse con menos rigor, y si no con autonomía, mucho más libremente.

Occidente era "decadente", y a través de un Spengler profusamente leído y resemantizado, se abría un espacio para la consideración de la originalidad latinoamericana, de su novomundismo y sus posibilidades futuras. La juventud de América Latina se recortaba sobre la vejez y agotamiento de la "vieja Europa". La Revolución Soviética y la Revolución Mexicana mostraban la vitalidad de sociedades no clásicamente europeas que trazaban vigorosas formas de cambio. Por otra parte, el hospitalario México de José Vasconcelos, fue receptor de todos los exilios y albergue frecuente de aquellos intelectuales que pensaban los cambios.

Si estas eran las coordenadas ideológicas, otro tanto ocurría con las históricas: el tema antiimperialista (mejor, antinorteamericano), adelantado por José Martí, insinuado sublimadamente por Rodó y desarrollado por Ugarte, cobró una urgente centralidad en los años veinte al calor de la recia política del big stick del primer Roosvelt. El antiimperialismo como actitud y reflexión teórica y política, también contribuyó a recortar la identidad de un destino común latinoamericano. Es éste el escenario privilegiado de recepción de la invitación de los estudiantes cordobeses a "vivir una hora americana". Así, al paso de los divulgadores de la experiencia de Córdoba en la región (entre los que se cuentan figuras tan distintas, en un arco que va desde Alfredo Palacios hasta José $A_{r c e^{12}}$ ) sumado a la vocación concreta de las federaciones estudiantiles de compartir su experiencia con sus pares latinoamericanos, el discurso latinoamericanista de los reformistas se irá tiñiendo de contenidos y significados más precisos. Tras el imperativo de una explicación más introspectiva sobre las realidades de la región, no deja de advertirse la clara vocación dirigente de estos jóvenes, al tiempo que ese movimiento intelectual y político va cargándose de significados que trascienden las aulas universitarias. José Carlos Mariátegui escribía en los Siete Ensayos sobre el aporte de los movimientos estudiantiles a la renovación de ideas en América Latina:

El movimiento estudiantil que se inició con la lucha de los estudiantes de Córdoba, por la reforma de la universidad, señala el nacimiento de la nueva generación latinoamericana. El movimiento se presenta íntimamente ligado con la recia marejada posbélica. Las esperanzas mesiánicas, los sentimientos 
revolucionarios, las pasiones místicas propias de la posguerra, repercutían particularmente en la juventud universitaria de Latinoamérica (...). Todos convienen que este movimiento, que apenas ha formulado su programa, dista mucho de proponerse objetivos exclusivamente universitarios y en que, por su estrecha y creciente relación con el avance de las clases trabajadoras y con el abatimiento de los viejos privilegios económicos, no puede ser entendido sino como uno de los aspectos de una profunda renovación latinoamericana. (MARIÁTEGUI, 1978, p. 109-110)

La democratización de las unidades académicas en América Latina se enfrentó con gobiernos de carácter dictatorial y base estrecha, que evidenció la esterilidad de un reclamo sectorial y llevó al movimiento estudiantil a estable $\urcorner$ cer una relación con la sociedad y la política en la que la apertura, la democratización y modernización universitaria se fundía con la impugnación a los gobiernos oligárquicos y la búsqueda de salidas políticas emancipatorias. Partidos tales como el APRA peruano, Acción Democrática en Venezuela o la instalación del gobierno de Grau San Martín en Cuba están estrechamente ligados al reformismo estudiantil, primera escuela política de sus cuadros dirigentes. ${ }^{13}$

Esa construcción latinoamericana tuvo en el Congreso Internacional de Estudiantes, realizado entre el 20 de septiembre y el 8 de octubre en el México revolucionario, uno hito importante. Fue convocado y organizado por José Vasconcelos (rector de la Universidad Nacional Autónoma de México) y Daniel Cosío Villegas (flamante secretario de la Federación de Estudiantes Mexicanos). La iniciativa, en el año de Centenario de la independencia de México, se realizaba, no sin algunas tensiones, en el marco de la política pacificadora de Álvaro Obregón. Aun cuando las delegaciones no eran solo latinoamericanas, eran éstas más representativas de los estudiantes que los "delegados" de EEUU, Alemania, Noruega, China o Japón, estos últimos representados por diplomáticos con sede en México. ${ }^{14}$

Por razones de espacio y oportunidad, no desarrollaremos los entretelones de la organización del congreso ni sus posteriores derroteros. ${ }^{15}$ Queremos enfatizar, en principio, algunos tramos el contexto de las ideas que se reflejan en sus resoluciones y contemporáneamente destacar ese Congreso como un espacio de sociabilidades de larga data a la que hicimos referencia, con alguna trayectoria que consideramos relevante.

En principio la enunciación de los actores a nuestro juicio está en un espacio de intersección entre los sustantivos "juventud", "universitarios" y una no explícita por las características incipientes de su identificación: intelectuales. ${ }^{16}$ Las resoluciones llevan la marca de un idealismo ético, algo mesiánico, con notas iluministas y heroicas tributarias, entre otras influencias, de la figura intelectual del grupo grupo Clarte! que encabezaba Henri Barbusse y nucleaba a Emile Zola, Anatole France, Romain Rolland, entre otros. Esa revista inspiró “Claridades" en varios países de la región que fueron lugares de intercambio entre los miembros de las Universidades Populares, las izquierdas y/o vanguardias políticas y estéticas. Por ejemplo: el joven Pablo 
Neruda desde su Temuco natal era corresponsal de la revista Claridad, órgano de la Federación de Estudiantes de Chile. ${ }^{17}$

La Resolución primera del Congreso señala "La juventud universitaria proclama que luchará por el advenimiento de una nueva humanidad, fundada sobre los principios modernos de justicia en el orden económico y político." (FEDERACIÓN INTERNACIONAL DE ESTUDIANTES, 1922, p. 7) Desagregando el concepto general proponen: luchar por la abolición del Estado como expresión de la dominación "de los menos por los más", contra la "explotación del hombre por el hombre y la organización actual de la propiedad”, "la integración de los pueblos en oposición al principio patriótico del nacionalismo", la crítica al positivismo, declarando que "las ciencias morales y políticas queden fundadas sobre la coordinación armónica del pensar, el sentir y el querer como medios de explicación.” (p. 8) También se establece la creación y fortalecimiento de Universidades Populares "inspirando su acción en los modernos postulados de justicia social." La parte quinta de los resultados está dedicada a las relaciones internacionales ante el peligro de una nueva Guerra Mundial. La declaración genérica "las relaciones internacionales deben descansar sobre la integración de los pueblos en una comunidad universal" se desagrega en cuestiones devenidas de la crítica al Tratado de Versalles, la condena a las "las tendencias imperialistas y de hegemonía" y la "conquista territorial y todos los atropellos de fuerza" junto con el señalamiento de dos cuestiones regionales que marcaron una constante en la década: el repudio a las dictaduras (en este caso la de Juan Vicente Gómez en Venezuela), y la oposición al "avance imperialista que sobre Santo Domingo y Nicaragua está ejerciendo el gobierno de los Estados Unidos."(p. 9-10)

Consideramos que esta plataforma común instaló un piso de consensos entre las juventudes contestatarias de la región que sumado al Programa Máximo del APRA (fundado durante el exilio de Víctor Raul Haya de la Torre en México en $1924^{18}$ ) establecía un sendero de afinidades electivas entre las nuevas generaciones intelectuales y políticas que se extendieron y precisaron a través del prisma de las realidades continentales a lo largo de la década, postulados algunos que la trascenderán, independientemente de bifurcaciones y polémicas. Un ejemplo poco advertido es que tres Premios Nobeles de literatura latinoamericanos compartieron esa estructura de sensibilidad en los años veinte. Gabriela Mistral (Premio Nobel 1947) había llegado a México en julio de 1922 y desplegó una destacada tarea docente y literaria formando parte del fáustico programa educativo del "Maestro de la Juventud" José Vasconcelos, el guatemalteco Miguel Ángel Asturias (Premio Nobel 1967) asistió al Congreso Internacional de Estudiantes y, como se señaló, contemporáneamente Pablo Neruda (Premio Nobel 1971) escribía en la revista estudiantil Claridad.

Otro asistente al Congreso que establecería lazos indelebles a partir de la experiencia en el Congreso estudiantil de México fue el reformista platense Arnaldo Orfila Reynal. Su amistad con Daniel Cosío Villegas, Alfonso Reyes, Manuel Gómez Morín, Pedro Henríquez Ureña (con este último primero en México y luego durante su primera estancia en Argentina: 1921-1924), selló sociabilidades que cambiarían gran 
parte de la industria editorial de la región y también el campo cultural, intelectual y académico latinoamericano.

Orfila formó parte de las revistas ligadas a la Reforma Universitaria Renovación y Valoraciones, desde ésta última Pedro Henríquez Ureña "recomienza, la práctica de la escritura ensayística apenas radicado en la Argentina, pudiéndose leer en algunas de sus intervenciones líneas fundamentales de su pensamiento crítico, que más tarde se afianzan en sus más importantes contribuciones a la historia de la literatura latinoamericana." (SANCHOLUZ, 2013, p. 95) En las décadas del treinta y cuarenta Orfila Reynal fue corresponsal en la Guerra Civil Española de la revista socialista La Vanguardia, escribió para la Editorial Claridad de Antonio Zamora, entre otras actividades intelectuales. Tuvo poca suerte (o quizá una vocación estudiantil equivocada), en el ejercicio de su título en de Doctor en Química. Esa aparente frustración se saldó con la invitación a hacerse cargo de establecer en 1944 una filial de la editorial Fondo de Cultura Económica (FCE) en Buenos Aires. ${ }^{19}$ Esa idea surgió de la mediación de Henríquez Ureña y Alfonso Reyes con el director del FCE, Daniel Cosío Villegas (aquel secretario de la FEM que presidió el Congreso Internacional de Estudiantes) para ayudar a sortear su frágil inserción laboral. La "Casa de la Cultura Mexicana en Argentina" se inauguró el 1 de enero de 1945. Tres años después fue convocado para hacerse cargo de la dirección del FCE en México cuando Cosío Villegas se retira transitoriamente para escribir su fundamental Historia Moderna de México con una beca de la Fundación Rockefeller. A partir de la renuncia de Cosío en 1952, Orfila asume la dirección de FCE.

En los años cuarenta la editorial había alcanzado notoriedad
continental como productora de traducciones imprescindibles
para la renovación de las Ciencias Sociales [Weber, Marx,
Mannheim, Keynes, Dobb, entre otros clásicos] y como medio
de expresión del ensayo sobre los problemas de América. En los
primeros años bajo la dirección de Orfila el catálogo se abrió en
dos marcadas direcciones: la promoción de obras literarias de
autores mexicanos y la búsqueda del lector masivo que surgía
gracias a la ampliación de la alfabetización, de la matrícula
universitaria y a la profesionalización de las Ciencias Sociales
(SORA, 2017, p. 69)

Esa gestión retoma y profundiza las líneas editoriales de Cosío Villegas. Las colecciones se multiplican en número y en importancia: Extremos de América, Tezontle y fundamentalmente Breviarios y Letras Mexicanas. A México acuden exiliados políticos de Guatemala, Cuba, Chile, Venezuela, Ecuador, y muchos de ellos trabajan en el FCE como correctores y (...) con Letras Mexicanas se formaliza el reconocimiento de la gran literatura que en el país se produce" (MONSIVAIS, 1997). Una nueva generación de escritores (Juan Rulfo, Carlos Fuentes, Rosario Castellanos, entre otros) publicaron sus primeros libros en el Fondo.

Fue tal el impacto de actualización, difusión, modernización y proyección latinoamericana que no llama la atención que en los Congresos de escritores e 
intelectuales latinoamericanos, tan frecuentes en la década de 1960, se apelara a la editorial para sortear uno de los obstáculos más tematizados (hasta hoy día) en los congresos latinoamericanos: superar el aislamiento y el desconocimiento de la producción latinoamericana Así, las referencias político-literarias más significativas del campo intelectual de los años sesenta se dirigen a Orfila Reynal en el Congreso de Intelectuales en la Universidad de Concepción (Chile, 1962): "Elevamos ante usted la siguiente sugestión: crear, dentro de la estructura editorial del Fondo, una colección popular de escritores iberoamericanos como un arma efectiva, capaz de influir decisivamente en el crecimiento de nuestra expresión y de darle la divulgación más amplia y fecunda (...)." (ALBURQUERQUE, 2000, p. 343)20

Hacia 1965 una campaña maccartista a partir de una denuncia de la Sociedad Mexicana de Geografía y Estadística, acusó a Orfila de "traición a la patria” durante el gobierno de Gustavo Díaz Ordaz. ${ }^{21}$ "La reacción al cese diazordacista de Orfila fue inmediata y constituye una de las páginas más hermosas de la vida cultural mexicana. Todos los amigos (y deudores) de Orfila abandonamos el Fondo de Cultura Económica. Elena Poniatowska tomó la iniciativa de crear una nueva editorial y prestó para ello su propia casa como oficina. Así nació el Siglo XXI, Orfila pudo continuar su extraordinaria tarea editorial y sus amigos encontramos un nuevo hogar para nuestros libros."(FUENTES, 1998) Siglo XXI publica las mayores contribuciones del campo intelectual, cultural y político latinoamericano marcado por el giro de la Revolución Cubana: el boom de la narrativa, la teoría de la dependencia, la Teología de la Liberación, revisiones del marxismo. Publica a Pablo González Casanova, a Paulo Freire, Nikos Poulantzas, a Jackes Lacan, los revolucionarios centroamericanos, los clásicos del marxismo.

Entre FCE y Siglo XXI, Orfila fue llamado en 1957 por el entonces rector de la Universidad de Buenos Aires, Risieri Frondizi, para asesorarlo en la creación de una editorial universitaria. Orfila estuvo tres meses en Buenos Aires y dejó diseñada EUDEBA en sus trazos más pormenorizados, desde las colecciones a las series, desde la estructura comercial al precio de los libros, escogiendo también a su director, el editor Boris Spivacow. Por entonces, el vicerrector de la UBA era aquel estudiante reformista José Babini, quien se convirtió en el primer presidente del Directorio de la flamante editorial. ${ }^{22}$

Esta apretada síntesis de la trayectoria de quien viviera cien años y los llenara de libros, intenta recuperar esas sociabilidades estudiantiles y las herencias y genealogías del reformismo como movimiento político-cultural del pensamiento latinoamericano.

Sería un laberinto muy difícil de sortear haber cursado una carrera de humanidades o de ciencias sociales en América Latina o ser un curioso lector de narrativa o temas contemporáneos sin haber leído algún libro de FCE, Siglo XXI o EUDEBA.

Creencias y trascendencias del movimiento reformista en América Latina. La actualización de esta tradición a lo largo de un siglo se encuentra en constante tensión con su movimiento opuesto: el de su cristalización en frases antes vigorosas, repetidas en ocasiones como un mantra anestésico cuando no conformista. Exclaustración de la cultura, humanismo, extensión universitaria, cogobierno, libre ingreso, laicismo, 
creación de conocimiento crítico, solidaridad latinoamericana, son herencias del movimiento reformista. Camino no lineal de construcción colectiva ya que es dable advertir que mucho de esos postulados fueron discutidos y aún suspendidos en los años veinte y durante los seis golpes militares de la historia argentina desde 1930. Pero allí nos quedan las resonancias de un movimiento cuya vitalidad quizá resida en lo inacabado. En el número de julio de 1936 de la Revista Flecha, Deodoro Roca impulsaba una encuesta sobre el movimiento estudiantil de la Reforma bajo el título Punto Final y lanzaba esa flecha hacia el futuro: “¿De "lo que fue”, sabemos más que antes? No es probable. Para nosotros es lo que pugna por ser, lo que queremos que sea, lo que será." (ROCA, 1936, p. 3)

\section{Referências}

ALBURQUERQUE FUSCHINI, G. La red de escritores latinoamericanos en los años sesenta: Universum, n. 15, 2000, p. 337-350.

ÁLVAREZ, G. Los cincuenta y nueve días que proyectaron sesenta años de edición universitaria. En ÁLVAREZ, G, ARCHAIN, A, DÍAZ, C. Un editor de tres siglos. La vida y los libros de Arnaldo Orfila Reynal, Buenos Aires: EUDEBA, 2015, p. 35-43.

ARCHIVO ORAL DE LA UNIVERSIDAD DE BUENOS AIRES (AOUBA). Entrevista a Miguel Murmis. MFN:84. Abril 1988.

BARROS E. Declaraciones del Señor Enrique Barros, presidente de la Federación Universitaria de Córdoba: La Nación, Buenos Aires, 10 de julio de 1918.

BIAGOSH, E. Universidad y Democracia. En DEL MAZO, G. La Reforma Universitaria. El Primer Congreso Nacional de Estudiantes Universitarios (Córdoba, 1918), Buenos Aires: Publicaciones del Circulo Medico Argentino y Centro de Estudiantes de Medicina, T. III, 1927, p. 100-131.

BUSTELO, N. La reforma universitaria desde sus grupos y revistas: Una reconstrucción de los proyectos y las disputas del movimiento estudiantil porteño de las primeras décadas del siglo XX (1914-1928). Tesis de posgrado. Universidad Nacional de La Plata. Facultad de Humanidades y Ciencias de la Educación. En Memoria Académica. Disponible en: http://www.memoria.fahce.unlp.edu.ar/tesis/te.1307/te.1307.

CLARK, B. El sistema de educación superior. México: Nueva Imagen/ UAM, 1983.

DEL MAZO, G. Estudiantes y gobierno universitario, Buenos Aires: El Ateneo, 1946.

DERRIDA, K. La filosofía como institución. Barcelona: Granica. 1984.

FEDERACIÓN INTERNACIONAL DE ESTUDIANTES. Resoluciones del Congreso Internacional de Estudiantes de México, México, 1922. 
FRANCO, J. La cultura moderna en América Latina. México: Grijalbo. 1985.

FUENTES, C. Cien años de Orfila Reynal: El País, 16 de enero de 1998. https://elpais. com/diario/1998/01/16/cultura/884905207_850215.html consultado el 23 de mayo de 2018.

FUNES P. La Facultad de Filosofía y Letras. Creación e Institución (1896-1930): Anacronismo e Irrupción: Revista de Teoría y Filosofía Política Clásica y Moderna, Buenos Aires, n. 14, v. 8, p. 180-202, 2018.

FUNES, P. Salvar la Nación. Intelectuales, cultura y política en los años veinte Latinoamericanos. Buenos Aires: Editorial Prometeo, 2006.

HAYA DE LA TORRE, V. R. El antiimperialismo y el APRA. Santiago de Chile: Ercilla, 1936. HENRÍQUEZ UREÑA, P. La Utopía de América. Caracas: Biblioteca Ayacucho. 1978.

INGENIEROS, J. La Revolución universitaria se extiende ya por toda la América Latina". En DEL MAZO, G.: La Reforma Universitaria. Buenos Aires: Publicaciones del Círculo Medico Argentino y Centro de Estudiantes, de Medicina, T. VI, p. 379-380. Originalmente escrito como editorial en la Revista Renovación, marzo de 1924.

INGENIEROS, J. La universidad del porvenir y otros escritos sobre Filosofía, Educación y Cultura. Buenos Aires: Círculo Médico y Centro de Estudiantes de Medicina, 1920.

LA GACETA UNIVERSITARIA, Año I, 1918.

MARIÁTEGUI, J. C. Siete Ensayos de interpretación de la realidad peruana. México: ERA, 1978.

MONSIVÁIS, C. En el centenario de Arnaldo Orfila Reynal: Proceso. México, 19 de julio de 1997. https://www.proceso.com.mx/176035/en-el-centenario-de-arnaldo-orfila-reynal.

NERUDA, P. Confieso que he vivido. Memorias. Buenos Aires: Losada, 1974.

ORTEGA Y GASSET, J. El tema de nuestro tiempo, Buenos Aires: Espasa Calpe, 1947. PORTANTIERO, J. Estudiantes y Política en América Latina. México: Siglo XXI, 1978.

ROCA, D. Discurso leído en la sesión de clausura del Primer Congreso Nacional de Estudiantes universitarios en Córdoba. 30 de julio de 1918: Ideas, n. 8, julio 1918. 301-315.

ROCA, D. Punto Final: Flecha, n. 16, 21 de julio de 1936, p. 1-5.

ROJAS, R. La Universidad de Tucumán. Tres conferencias. Buenos Aires: Librería Argentina de Enrique García, 1915.

SANCHOLUZ C. Desplazamiento y nuevos arraigos: Pedro Henríquez Ureña y la revista platense Valoraciones: Anales de Literatura Hispanoamericana, vol. 42, 2013, p. 91-105.

SORA, G. Editar desde la izquierda en América Latina. La agitada historia del Fondo de Cultura Económica y de siglo XXI, Buenos Aires: Siglo XXI, 2017. 
SUASNÁBAR, C. La Reforma Universitaria de Córdoba de 1918, una mirada histórica de la relación entre universidad, intelectuales y política. En Práxis Educativa, Ponta Grossa, n. 1, v. 4, p. 51-61, jan-jun, 2009.

TERÁN, O. José Ingenieros. Antiimperialismo y Nación. México: Siglo XXI, 1979.

WATSON, G. "Teoría del Gobierno Republicano de la Universidad”. En DEL MAZO, G. La Reforma Universitaria 1918-1926. Buenos Aires: Círculo Médico y Centro de Estudiantes de Medicina, 1927, T.I p. 26-39.

YANKELEVICH, P. Estamos pisando sobre una revolución. México y el reformismo universitario argentino de 1918: Argumentos, revista de crítica social, Buenos Aires, n. 20, octubre 2018. p. 158-180. Disponible en: http://publicaciones.sociales.uba.ar/ index.php/argumentos/index

YANQUELEVICH, P. Miradas australes. Propaganda, cabildeo y proyección de la Revolución Mexicana en el Río de la Plata, 1910-1930. México: Instituto de Estudios Históricos de la Revolución Mexicana-Secretaría de Relaciones Exteriores. 1997.

\section{Notas}

1 Sobre esos tres niveles de análisis véase CLARK, 1983

2 José Nicolás Matienzo fue ministro de la Suprema Corte de la provincia de Buenos Aires, responsable del Departamento Nacional del Trabajo de la Nación en la presidencia de José Figueroa Alcorta, Procurador general de la Nación en la presidencia de Hipólito Yrigoyen quien lo envió como interventor a la Universidad Nacional de Córdoba en apoyo a los reclamos estudiantiles de 1918. Rodolfo Rivarola fue juez, Secretario de la Suprema Corte de Justicia de la Provincia de Buenos Aires en el período de 1889 a 1893, Fiscal de Cámara en el lapso de 1893 a 1898 y simultáneamente Vocal del Consejo de Educación de la Provincia de Buenos Aires. Fue Presidente de la Universidad Nacional de la Plata en el período 1918-1920. Fundó y dirigió durante muchos años la Revista Argentina de Ciencias Políticas.

${ }^{3} \mathrm{Al}$ respecto véase: FUNES, 2018.

4 "Hay familias que tienen hasta diez representantes en la universidad, partidos políticos que cuentan con un altísimo porcentaje de afiliados; y en cuanto a la religión ésta tiene el noventa y nueve por ciento sometido a la más escrupulosa selección y vigilancia”. (BARROS, 1918, p. 11).

5 La Universidad Nacional del Litoral se creó sobre la Universidad provincial de Santa Fe (1889) y fue nacionalizada en 1919 abarcando escuelas e institutos asentados en las ciudades de Santa Fe, Paraná, Rosario y Corrientes. En el caso de la Universidad Nacional de Tucumán su nacimiento inspirado en el proyecto de Juan B. Terán proponía explícitamente evitar la réplica de las universidades existentes en el país. Bajo la tríada "regional, técnica y pragmática", sus fundamentos eran arraigar los estudios superiores a las necesidades agrarias, biológicas, industriales de la región del Noroeste Argentino.

6 Las citas del Manifiesto son tomadas del documento original: La Gaceta Universitaria, Número extraordinario, Año 1, N 10, 21 de junio de 1918, s/f. 
7 Sobre la Asociación Córdoba Libre y las revistas que inspiraron a la generación reformista, véase: BUSTELO, 2015, p. 97 y ss.

8 La Corda Frates era una logia conservadora y ultramontana que tenía el control académico y político de la Universidad Nacional de Córdoba y de gran parte de los poderes públicos de la provincia de Córdoba. "La Corda, esa hidra monstruosa que desorganizó el partido radical, absorbió la Municipalidad, que se impuso en el Consejo de Educación, que dominó el Poder Ejecutivo mismo (...) La Corda que se adueñó de algunos puestos del Poder Judicial, que merodea todos los partidos políticos (...) esa grotesca masonería con el escapulario al pecho y diatribas en los diarios, está en todas partes”. (LA GACETA UNIVERSITARIA, 1918)

${ }^{9}$ El primer Congreso Nacional de Estudiantes tuvo lugar en Córdoba en el mes de julio de 1918, con 65 representantes de las cinco 5 universidades. El encargado de informar las resoluciones de la comisión sobre el régimen de gobierno fue el delegado de la Federación Universitaria de Buenos Aires, Guillermo Watson. (WATSON, 1926, p. 29)

${ }^{10}$ Es dable advertir, además, el encono personal de Ingenieros respecto al presidente Sáenz Peña, ya que le había negado en 1911 la Cátedra de Medicina Legal de la Universidad de Buenos Aires.

11 "Nuestra plutocracia educa muñecas de Paris, la burguesía mujeres igualmente artificiales (técnicas de piano, de deshilvanada lectura y también esclavas de la última moda de la Rue de la Paix). Unas y otras encaminadas exclusivamente al matrimonio (...). La clase asalariada, produce eternas cenicientas; estas llegan por excepción a la escuela media (...) y resulta que la inmensa mayoría recibe una enseñanza inadecuada para su sexo. Ni en éstas ni en aquellas existe el más ligero asomo del ideal de mujer que esta democracia necesita." (BIAGOSH, 1927, p. 115).

12 Es conocido el fuerte efecto que produjeron las conferencias de Alfredo Palacios (1919) entre los estudiantes de la Universidad de San Marcos en Lima. Este fue un importante punto de partida para la reorganización del movimiento estudiantil peruano, para la creación de la Universidad Popular González Prada y también para el liderazgo de las jornadas de protesta obrero-estudiantil en 1923 lideradas por el Presidente de la FEP, Víctor Raúl Haya de la Torre, figura que tendrá una gran influencia en la difusión y reelaboración de los postulados reformistas. Menos conocida es la difusión del movimiento reformista argentino por parte del conservador José Arce en Cuba hacia fines del año 1922. El Dr. Arce, en calidad de rector de la UBA, pronunció una serie de conferencias en La Habana que tuvieron honda repercusión entre los estudiantes cubanos: "su presencia y elocuencia actuaron como un verdadero detonador en aquel medio". Al respecto puede verse un testimonio personal en: SOTO, 1979, p. 108 y ss.)

13 Un hilo de Ariadna filia la lucha antimachadista, el protagonismo de la Federación de estudiantes cubanos y la Revolución Cubana, como lo expresara el libro de Juan Carlos Portantiero (1978) escrito al calor de las rebeliones estudiantiles de los años sesenta.

${ }^{14}$ La delegación mexicana estuvo compuesta por Cosío Villegas, Brito Foucher, Raúl J. Pous Ortiz, Miguel Palacios Macedo y Francisco del Río Cañedo. Se sumaron a ella Manuel Gómez Morín, Vicente Lombardo Toledano, Octavio Medellín Hostos, Jorge Prieto Laurens y Ramón Beteta Quintana. Por Argentina asistieron Ripa Alberdi, Gabriel del Mazo, Miguel Bonchil, Enrique Dreyzin, Pablo Vrieland. Arnaldo Orfila Reynal. La delegación peruana estaba compuesta por Erasmo Roca y Raúl Porras Barrenechea, y se les unió un invitado especial de Vasconcelos: Víctor Andrés Belaúnde. Entre los demás latinoamericanos estaban el cubano Eduardo Betancourt; los nicaragüenses Gustavo Jerez Tablada, Guillermo J. Maritano y Salomón de la Selva (éste ejercía como diplomático en Estados Unidos); los costarricenses Antonio Zelaya Castillo y Óscar Vargas; los guatemaltecos Miguel Ángel Asturias, Carlos Zamayoa y Óscar Humberto Espada; el dominicano Pedro Henríquez Ureña fue en representación de la Universidad de Minessota; los hondureños Roberto Barrios y Rafael Heliodoro Valle, ambos residían en México. 
${ }^{15} \mathrm{Al}$ respecto véase: Yankelevich (2015).

16 Sobre las autoidentificación en términos de "intelectuales" de los hombres de letras en la década de 1920, véase Funes (2006) p.39-69.

17 "Yo había sido en Temuco el corresponsal de la revista Claridad, órgano de la Federación de Estudiantes (...). Las noticias que en el año 1920 nos llegaron a Temuco marcaron a mi generación con cicatrices sangrientas. Las juventud dorada', hija de la oligarquía, había asaltado y destruido el local de la Federación de Estudiantes. Domingo Gómez Rojas, joven esperanza de la poesía chilena enloqueció y murió torturado en un calabozo. La repercusión de este crimen (...) fue tan profunda y vasta como habría de ser el asesinato en Granada de Federico García Lorca” (NERUDA, 1974, p. 52)

18 Los cinco puntos del programa máximo de la Alianza Popular Revolucionario Americana: “1. Acción contra el imperialismo yanqui. 2. Por la unidad política de América Latina. 3. Por la nacionalización de tierras e industria. 4. Por la internacionalización del Canal de Panamá. 5.Por la solidaridad con todos los pueblo y clases oprimidas del mundo." (HAYA DE LA TORRE, 1936, p. 35).

${ }_{19}$ Fondo de Cultura Económica se creó en 1934 junto con la Revista El Trimestre Económico, en principio con el objetivo de fortalecer las políticas públicas sobre todo en la formación económica de los cuadros del Estado y su actualización en los debates económicos a partir de la crisis económica mundial junto con una editorial que tradujera las principales obras del tema. Ambas con un financiamiento estatal a través de un fideicomiso que no intervenía en las decisiones editoriales. Desde esos iniciales objetivos el catálogo se amplió temáticamente hacia las ciencias sociales y las humanidades en México y América Latina. El Fondo editó Economía, Sociología, Historia, Filosofía, Política y Derecho, Letras y los libros de El Colegio de México. Se destacan en las humanidades las colecciones Tierra Firme y Biblioteca Americana. Desde su creación hasta 1945 fue dirigida por Daniel Cosío Villegas.

20 Firman: Héctor P. Agosti, José Bianco (Argentina); Jesús Lara, Gil Imana Garrón, Nilda Núñez del Prado (Bolivia); Thiago de Mello, Carolina María de Jesús (Brasil); Gerardo Molina, (Colombia); Alejo Carpentier (Cuba); Benjamín Carrión (Ecuador); Carlos Fuentes (México); Augusto Roa Bastos (Paraguay); José María Arguedas, José Miguel Oviedo (Perú); Claribel Alegría (El Salvador); Mario Benedetti (Uruguay); Pablo Neruda; Gonzalo Rojas, Fernando Alegría, Braulio Arenas, Luis Oyarzún, Amanda Labarca, José Donoso, Jaime Valdivieso, Hernán San Martín, y Alfonso Echeverría (Chile). Casa de las Américas, № 11-12, marzo-junio, 1962. Alburquerque Fuschini, 2000, p. 343).

21El detonante fue la publicación Escucha yanqui, de Wright Mills (1960), y Los hijos de Sánchez de Oscar Lewis (1967). Al respecto véase SORÁ, 2017, p. 145-169.

22 Sobre la organización y creación de EUDEBA véase: ÁLVAREZ, 2015, p. 35-43. 
Patricia Funes es doctora en Historia. Investigadora del Consejo Nacional de Investigaciones Científicos y Técnicas (CONICET). Profesora titular de la materia Historia Social Latinoamericana da Carrera de Sociología y Profesora Asociada Titular de la Carrera de Ciencia Política, Facultad de Ciencias Sociales da Universidade de Buenos Aires. Sus investigaciones se refieren al campo de las ideas políticas y culturales en América Latina del siglo XX. Entre sus últimas publicaciones: Patricia Funes (directora), Revolución, dictadura y democracia. Lógicas militantes e militares na historia argentina no contexto latinoamericano. Imago Mundi, Buenos Aires, 2016. Patricia Funes, Historia mínima de las ideas políticas en América Latina, El Colegio de México / Turner, México / Madrid, 2014. 